\title{
Specialists Consulted
}

Robert Austerlitz (Uralic, Chukchi-Kamchatkan)

Yvonne Bastin (Bantu)

M. Lionel Bender (Nilo-Saharan, Afro-Asiatic)

Paul K. Benedict (Sino-Tibetan, Austric)

Richard Bergman (Ubangian)

D. N. S. Bhat (Dravidian)

Robert Binnick (Mongolian)

Henrik Birnbaum (Slavic)

Paul Black (Cushitic, Australian)

Robert Blust (Austronesian)

Nicholas Bodman (Tibeto-Burman)

Lee E. Bohnhoff (Duru)

Luc Bouquiaux (Adamawa, Ubangian)

William Bright (Hokan)

Breandán Ó Buachalla (Celtic)

Robbins Burling (Burmese-Lolo)

Wallace L. Chafe (Siouan,

Caddoan, Iroquoian)

Matthew Chen (Chinese)

Christos Clairis (Southern Andean)

Frans van Coetsem (Germanic)
James T. Collins (Maluku)

Bernard Comrie

(Chukchi-Kamchatkan)

+Warren Cowgill (Indo-Hittite)

Irvine Davis (Ge)

Gyula Décsy (Uralic)

Desmond Derbyshire (Carib)

Gérard Diffloth (Austroasiatic)

Søren Egerod (Sino-Tibetan)

Christopher Ehret (Cushitic)

Murray Emeneau (Dravidian)

Ronald E. Emmerick (Iranian)

Harold C. Fleming (Omotic)

Thomas Gamkrelidze (Caucasian)

Ives Goddard (Algonquian)

Morris Goodman (Nilo-Saharan)

George Grace (Oceanic)

Joseph H. Greenberg (world)

Austin Hale (Sino-Tibetan)

Kenneth Hale (Australian, Uto-Aztecan)

Robert A. Hall, Jr. (Romance)

Robert Harms (Uralic)

André Haudricourt

(New Caledonia) 
Richard Hayward (Cushitic)

Robert Hetzron (Afro-Asiatic)

Carleton T. Hodge (Afro-Asiatic)

Larry Hyman (Grassfields Bantu)

V. V. Ivanov (Balto-Slavic)

Philip N. Jenner (Mon-Khmer)

Robert B. Jones (Karen)

Terrence Kaufman (Mayan)

Mary Key (South America)

M. Dale Kinkade (Salish)

Michael Krauss (Eskimo-Aleut, Na-Dene)

John R. Krueger (Altaic)

Ronald Langacker (Uto-Aztecan)

Margaret Langdon (Hokan)

Donald Laycock (Indo-Pacific)

F. K. Lehman (Tibeto-Burman)

Winfred Lehmann (Indo-European)

Fang-kuei Li (Tai)

W. B. Lockwood (Indo-European)

Eugene Loos (Panoan)

Horace G. Lunt (Slavic)

D. N. MacKenzie (Iranian)

Yakov Malkiel (Romance)

Gabriel Manessy (Gur)

Colin Masica (Indic)

James Matisoff (Tibeto-Burman)

David McAlpin (Dravidian)

Curtis McFarland (Philippines)

Alexander J. Militarev (Berber)

Marianne Mithun (Iroquoian)

David Moody (Borneo)

William Moulton (Germanic)

Paul Newman (Chadic)
Brian Newton (Greek)

Kemp Pallesen (Sama-Bajaw)

F. R. Palmer (Cushitic)

Andrew Pawley (Austronesian)

Rebecca Posner (Romance)

Ernst Pulgram (Italic)

Lawrence Reid (Philippines)

Calvin Rensch (Oto-Manguean)

David S. Rood (Siouan)

William J. Samarin (Adamawa, Ubangian)

J. David Sapir (West Atlantic)

Thilo C. Schadeberg (Kordofanian)

William R. Schmalstieg (Baltic)

Gary Simons (Santa Isabel, Southeast Solomons)

William A. Smalley (Miao-Yao)

Kenneth D. Smith (Borneo)

David Stampe (Munda)

Stanley Starosta (Austroasiatic)

Susan Steele (Uto-Aztecan)

Robin Thelwall (Nubian, Daju)

Laurence Thompson (Salish)

Darrell T. Tryon (Austronesian)

Robert Underhill (Turkic)

William S.-Y. Wang (Chinese)

E. O. J. Westphal (Khoisan)

Kay Williamson (Benue-Congo)

Alfred Willms (Berber)

Dean Worth (Chukchi-Kamchatkan)

Norman Zide (Austroasiatic)

David Zorc (Philippines, Australian) 